\title{
Rejuvenating liver and pancreas through cell transdifferentiation
}

\author{
Fei Yi ${ }^{1}$, Guang-Hui Liu ${ }^{1,3}$, Juan Carlos Izpisua Belmonte ${ }^{1,2}$ \\ ${ }^{1}$ Gene Expression Laboratory, Salk Institute for Biological Studies, 10010 North Torrey Pines Road, La Jolla, California 92037, USA; \\ ${ }^{2}$ Center for Regenerative Medicine in Barcelona, Dr. Aiguader 88, 08003 Barcelona, Spain; ${ }^{3}$ National Laboratory of Biomacromol- \\ ecules, Institute of Biophysics, Chinese Academy of Sciences, Beijing 100101, China \\ Cell Research (2012) 22:616-619. doi:10.1038/cr.2012.33; published online 28 February 2012
}

Induced pluripotent stem cells (iPSCs) and the cell transdifferentiation technologies are providing powerful tools to generate patient-specific cells for research and therapeutic applications. Hepatocytes and pancreatic $\beta$ cells are two endoderm-derived cell types drawing much attention due to their indispensable physiological functions and strong association with various diseases. Recent advances in hepatocyte and $\beta$ cell transdifferentiation have provided valuable insights into how to regenerate and restore normal functions of liver and pancreas under pathological conditions.

The discovery of iPSCs in 2006 has revoked the commandment that cell differentiation and lineage specification is an irreversible process, hence fundamentally changing the way we comprehend and study cell fate determination. An ultimate goal of regenerative medicine is built on the passion to produce healthy human cells for cell therapies. Unlike human embryonic stem cells, which have many ethical concerns, both cell reprogramming and

Correspondence: Juan Carlos Izpisua Belmonte ${ }^{\mathrm{a}}$, Guang-Hui Liu ${ }^{\mathrm{b}}$

aE-mail: belmonte@salk.edu, izpisua@cmrb.eu

bE-mail: ghliu@ibp.ac.cn cell transdifferentiation methods utilize somatic cells from the patient's own body. This advantage brings foreseeable hope for the implementation of autologous cell therapy, and may bypass many immunorejection-related issues [1]. However, the strategy to generate functional cells by coupling patient-specific iPSCs and directed cell differentiation has innate risks. For example, the likelihood of contamination by undifferentiated pluripotent cells may bring the risk of teratoma formation after cell transplantation; also, the extensive cell proliferation required for efficient cell reprogramming and pluripotent cell differentiation may lead to accumulation of genetic abnormities favoring cell proliferation and survival [2]. Therefore, the cell transdifferentiation strategy, which often does not involve intensive cell proliferation, outstands as a feasible choice. In recent years, accumulating evidence has revealed a previously unexpected potential of somatic cells undergoing transdifferentiation to convert into various cell types, in either transcription factor- and/or cytokinedependent manner. The notion of cell transdifferentiation has fueled a new branch of stem cell research and demonstrated an artificial yet creative way of thinking and promoting regenerative medicine research. Moreover, cell transdifferentiation enables an alternative approach to establish disease-specific cells with desired genetic backgrounds, which would facilitate the study of many important diseases.

Liver and pancreas are two important organs developed from embryonic endoderm. They are in charge of many essential life functions so that failure of either one results in severe life-threatening events. Hepatocytes and pancreatic $\beta$ cells are the major functional units of the two organs. Till now, efficient treatments for rescuing the malfunction of these vital cells are still missing. Clinical studies have demonstrated that cell transplantation-based therapy can support and restore functions of failed liver and pancreatic islets. However, broad clinical and scientific applications of human hepatocytes and $\beta$ cells are facing serious limitations due to the shortage of natural cell resources. Also, the current inability to culture and expand human hepatocytes and $\beta$ cells in vitro with unimpaired physiological functions represents a major obstacle in the field. To this end, generation of functional hepatocytes and $\beta$ cells from abundant somatic cell sources is in urgent need and would provide an invaluable resource for cell therapy and drug development.

Because of the outstanding cell plasticity and accessibility, fibroblasts have been considered a prime resource 
in cell transdifferentiation studies. Recently, two groundbreaking studies reported that fibroblasts can be directly reprogrammed into functional hepatocyte-like cells with a combination of endodermal and hepatic transcription factors [3, 4]. Huang et al. [4] found that ectopic expression of Gata4, Hnfl $\alpha$ and Foxa3, combining with the inactivation of $\mathrm{p} 19^{\text {Arf }}$, directly converted mouse adult fibroblasts into induced hepatocyte-like cells (iHep); while in the article by Sekiya et al. [3], a similar screening strategy identified Hnf4 $\alpha$ plus either Foxa1/Foxa2/Foxa3 for converting mouse embryonic and adult fibroblasts into iHep. From both reports, the iHep exhibited typical hepatocyte morphology, gene expression and protein secretions. Interestingly, the iHep efficiently expanded in vitro, a property that is similar to mouse adult liver progenitors but not seen in mature hepatocytes. Accordingly, it is generally agreed that these iHep may represent incomplete hepatocyte specification or an immature stage of hepatocytes. More importantly, iHep generated from both groups were able to pass the stringent in vivo engraftment test and repopulate up to $80 \%$ of the liver in fumarylacetoacetate hydrolase (Fah)-deficient mice [4]. Optimistically, it would be exciting if human iHep can be generated in the next step. However, the innate differences between mouse and human hepatocyte development may bring barriers of finding a common code for human iHep. Although the forced expression of one cocktail could generate mouse iHep, the same combination might not work as efficiently in human cells. Indeed, even in mouse iHep induction, the overall conversion efficiency could be as low as $0.3 \%$ from the starting population [3]. Therefore, even if one-cocktail induction of human iHep is possible, a more elaborate and multi-step method will likely to be needed to ensure the efficiency and quality of human iHep, and thus fully open the great potential of iHep in both research and clinic.
Mesenchymal stem cells (MSC) are another promising autologous source for regenerative medicine study and have been a hot topic in hepatocyte transdifferentiation. Though constantly under debate, there are accumulating evidences over the years supporting the hepatic transdifferentiation from MSC of different origins [5-8]. These hepatocyte-like cells transdifferentiated from MSC closely resemble hepatocyte characteristics in vitro. More importantly, they can also engraft into the parenchyma of the liver and at least partially restore liver functions in injury models in vivo [9].

The pancreas is a dual-function organ composed of different endocrine and exocrine cells. Among different types of pancreatic cells, the insulinproducing $\beta$ cell is of the most interest due to its tight physiological connection to diabetes. While both type I and type II diabetes can be effectively treated by insulin administration, the best way to cure diabetes is to restore the $\beta$ cell population. Unlike hepatocytes that have stem cell-like regenerative capability, the adult $\beta$ cells have very limited regenerative ability, which is insufficient to compensate the cell loss in diabetes. Because pancreatic exocrine cells and endocrine $\beta$ cells are closely related lineages in development and derived from common progenitors, conversion between them may require less epigenetic changes thus representing an appealing source of $\beta$ cell regeneration. Indeed, Melton and his colleagues reported that re-activation of three transcription factors (Ngn3, Pdx 1 and Mafa) in vivo reprogrammed pancreatic exocrine cells into endocrine $\beta$ cells in adult mice [10]. The authors utilized a cell tracking system and demonstrated that overexpression of the three key pancreatic factors converted acinar cells to insulin-producing $\beta$ cells with efficiency up to $20 \%$ in vivo [10]. These induced $\beta$ cells were indistinguishable from endogenous islet $\beta$ cells and expressed all key $\beta$ cells markers. Moreover, induced $\beta$ cells rescued mice from streptozotocin-induced diabetes, and remained functional up to three months [10]. This proof-of-principle study argued the plasticity of exocrine to endocrine transition in pancreas, and provided a theory basis for future investigation. More recently, the same strategy has been employed in an in vitro model. Akinci et al. [11] investigated the conversion of pancreatic exocrine cells into $\beta$ cells in vitro using adenovirus mediated Ngn3, Pdx1 and Mafa. The authors successfully generated induced $\beta$ cell-like cells with many shared properties. However, the induced $\beta$ cells failed to fully recapitulate the sensitivity of endogenous $\beta$ cells in response to glucose levels, which implies an optimal condition of the transdifferentiation is still missing and needs further investigation.

It has been debated for years that whether the massive loss of $\beta$ cells can be compensated from precursors or other heterologous sources. These transdifferentiation phenomena, if they exist, may happen in rare occasions and the initiating cells in which the conversion occurs are difficult to track. In 2010 , these challenges were overcome by Thorel et al. [12] using a nicely designed tractable mouse model. Thorel and colleagues generated a transgenic mouse model in which more than $99 \%$ of endogenous $\beta$ cells were chemically destroyed. Then using a $\beta$ cell tracking system, the authors clearly observed increased $\beta$ cell mass over time and demonstrated that new $\beta$ cells arose from healthy $\alpha$ cells through transdifferentiation [12]. Thorel and colleagues' discovery is very fascinating, as it revealed a previously unappreciated flexibility of cell fate conversion of endocrine cells. Also, the authors' method is different from the conventional cell transdifferentiation strategy, which often involves virus particle-mediated gene induction. Instead, the authors created a fatal situation of $\beta$ cell shortage, thereby maximally boosting the inherent plasticity of endocrine $\alpha$ cells. 


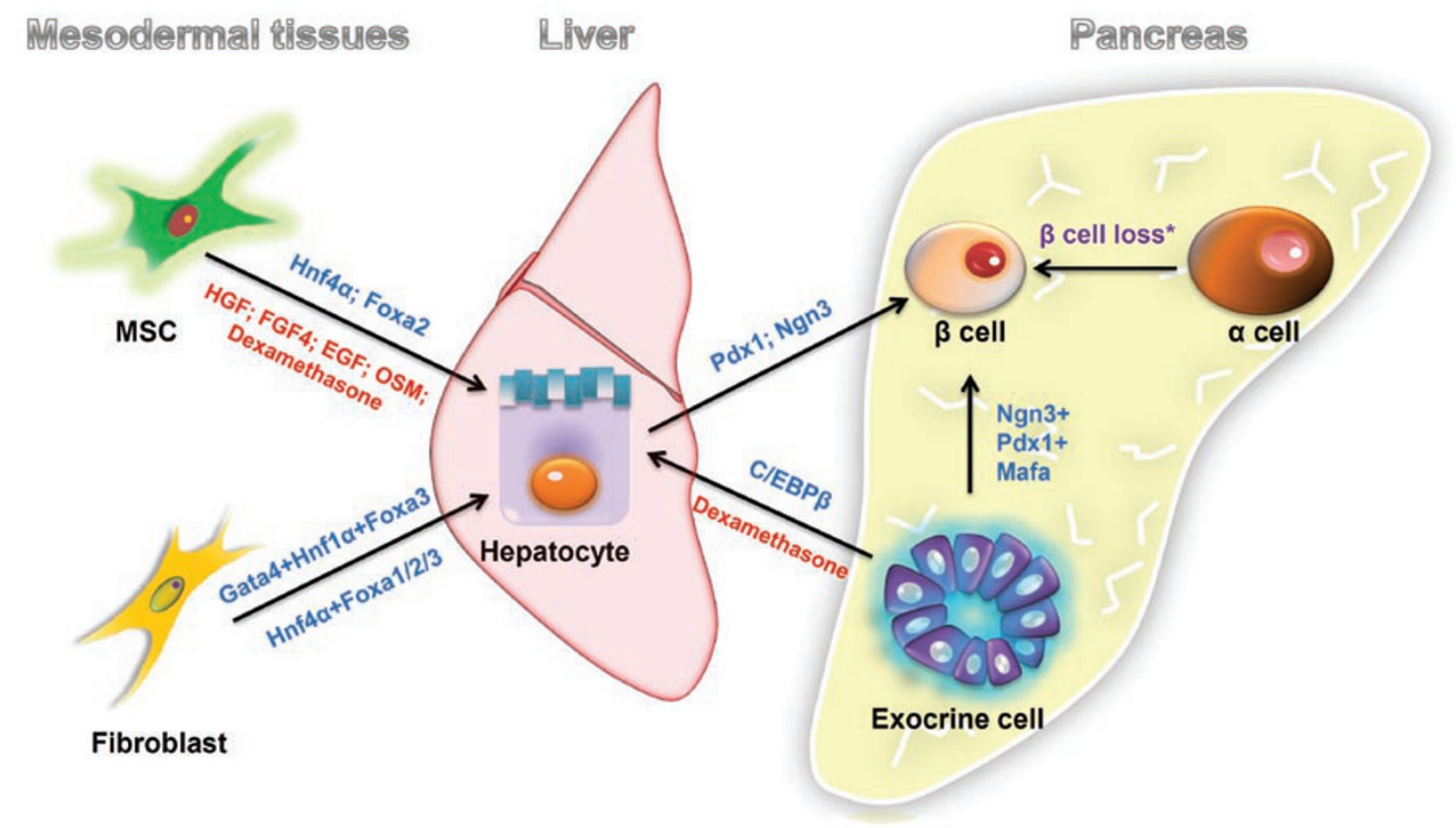

Figure 1 Summarization of reported cell transdifferentiation towards hepatocytes and pancreatic $\beta$ cells. Cell-fate-determining transcription factors are indicated in blue; inductive cytokines and small molecules are indicated in red. ${ }^{*}$ Transdifferentiation happened under physiological condition in vivo.

Although converted $\beta$ cells seemed to be frequently bifunctional, secreting both insulin and glucagon, which indicated an incomplete cell conversion [12], this study significantly extended our knowledge of pancreatic cell plasticity and regeneration.

Since liver and pancreas arise from the same bi-potential precursors in the anterior endoderm, it is reasonable to speculate that two closely related tissues may be inter-convertible. Specifically, it was believed that cells with properties similar to hepatic oval cells (a proposed hepatic progenitor population) also reside in the pancreas, which could transdifferentiate into functional hepatocytes upon transplantation. Glucocorticoid dexamethasone could efficiently induce rat pancreatic exocrine cells into hepatocytes [13]. Similarly, another report confirmed the inductive role of dexamethasone in transdifferentiation of pancreatic acinar cells into functional hepatocytes through a transient suppression of Wnt signaling [14]. These findings may suggest that adult pancreatic cells might retain epigenetic memory from their common embryonic origin with hepatocytes and thereby are flexible to convert into hepatocytes under permissive conditions. It is interesting to see that the switch between hepatocytes and endocrine $\beta$ cells is also feasible in a reciprocal manner. There have been reports of cell transdifferentiation of hepatocytes into pancreatic $\beta$ cells involving several key pancreatic factors. Among them, Pdx1 seems to play a key role in the hepatic-pancreatic cell fate conversion. In mouse, it has been reported that Pdx1 expression could lead to the conversion of hepatocytes into a pancreatic $\beta$-like cells that secret insulin $[15,16]$. On the other hand, it has also been found that the combination of $\mathrm{Pdx} 1$ and $\mathrm{Ngn} 3$ could synergistically induce expression of $\beta$ cell factors and insulin biosynthesis in liver, and drastically ameliorated glucose tolerance [17]. Interestingly, a study from Yechoor et al. [18] reported that the forced expression of Ngn3 alone failed to transdifferentiate mature hepatocytes but was com- petent in converting hepatic progenitor cells into a functional pancreatic $\beta$ cell lineage. Taken together, these studies demonstrate that liver and pancreatic cells may possess the plasticity of converting into each other under certain circumstances. Future studies aimed at the elucidation of the mechanism behind this metaplasia between these two essential endodermal lineages will help us to better understand and decipher the code necessary for liver and pancreas regeneration.

In summary, recent advances of liver and pancreatic cell transdifferentiation (Figure 1) are exciting indeed and have truly ignited new hopes for studying and treating many liver- and pancreasrelated diseases. Though many of them may not make an instant breakthrough in the clinic, they contribute enormously to our understanding of cell lineage determination and therefore may eventually lead to the success of cell therapies. While foreseeing great potentials of these new discoveries, certain issues will still need to be addressed within 
the coming years, such as the efficiency of the cell transdifferentiation, the maturation of the "hepatocyte- or $\beta$ celllike" cells, and the elimination of any possible tumor-initiating cells resulted from incomplete epigenetic conversion. Once we achieve a comprehensive understanding of the mystery behind cell transdifferentiation and are able to use the knowledge to refine and control the cell transdifferentiation process, we may be able to move from the bench to the clinic.

\section{Acknowledgments}

Work in the laboratory of JCIB was supported by Sanofi-Aventis, The Helmsley Charitable Trust and The Ellison Medical Foundation. GHL was supported by 100 Talents Program of the Chinese Academy of Sciences.

\section{References}

1 Jopling C, Boue S, Izpisua Belmonte JC. Dedifferentiation, transdifferentiation and reprogramming: three routes to regeneration. Nat Rev Mol Cell Biol 2011; 12:79-89.

2 Pera MF. Stem cells: The dark side of induced pluripotency. Nature 2011; 471:46-47.

3 Sekiya S, Suzuki A. Direct conversion of mouse fibroblasts to hepatocyte-like cells by defined factors. Nature 2011; 475:390-393.

4 Huang P, He Z, Ji S, et al. Induction of functional hepatocyte-like cells from mouse fibroblasts by defined factors. Nature 2011; 475:386-389.

5 Lee KD, Kuo TK, Whang-Peng J, et al. In vitro hepatic differentiation of human mesenchymal stem cells. Hepatology 2004; 40:1275-1284.

6 Chien CC, Yen BL, Lee FK, et al. In vitro differentiation of human placenta-derived multipotent cells into hepatocyte-like cells. Stem Cells 2006; 24:1759-1768.

7 Chen ML, Lee KD, Huang HC, et al. HNF-4alpha determines hepatic differentiation of human mesenchymal stem cells from bone marrow. World J Gastroenterol 2010; 16:5092-5103.

8 Ishii K, Yoshida Y, Akechi Y, et al. Hepatic differentiation of human bone marrow-derived mesenchymal stem cells by tetracycline-regulated hepatocyte nuclear factor 3 beta. Hepatology 2008; 48:597-606.

9 Banas A, Teratani T, Yamamoto Y, et al. Adipose tissue-derived mesenchymal stem cells as a source of human hepatocytes. Hepatology 2007; 46:219-228.

10 Zhou Q, Brown J, Kanarek A, Rajagopal J, Melton DA. In vivo reprogramming of adult pancreatic exocrine cells to beta-cells. Nature 2008; 455:627632.

11 Akinci E, Banga A, Greder LV, Dutton JR, Slack JM. Reprogramming of pancreatic exocrine cells towards a beta cell character using Pdx1, Ngn3 and MafA. Biochem J 2011; 442:539-550.

12 Thorel F, Nepote V, Avril I, et al. Conversion of adult pancreatic alpha-cells to beta-cells after extreme beta-cell loss. Nature 2010; 464:1149-1154.

13 Al-Adsani A, Burke ZD, Eberhard D, et al. Dexamethasone treatment induces the reprogramming of pancreatic acinar cells to hepatocytes and ductal cells. PLoS One 2010; 5:e13650.

14 Wallace K, Marek CJ, Hoppler S, Wright MC. Glucocorticoid-dependent transdifferentiation of pancreatic progenitor cells into hepatocytes is dependent on transient suppression of WNT signalling. J Cell Sci 2010; 123:21032110.

15 Ferber S, Halkin A, Cohen $\mathrm{H}$, et al. Pancreatic and duodenal homeobox gene 1 induces expression of insulin genes in liver and ameliorates streptozotocin-induced hyperglycemia. Nat Med 2000; 6:568-572.

16 Aviv V, Meivar-Levy I, Rachmut IH, Rubinek T, Mor E, Ferber S. Exendin-4 promotes liver cell proliferation and enhances the PDX-1-induced liver to pancreas transdifferentiation process. $J$ Biol Chem 2009; 284:33509-33520.

17 Kaneto H, Nakatani Y, Miyatsuka T, et al. PDX-1/VP16 fusion protein, together with NeuroD or Ngn3, markedly induces insulin gene transcription and ameliorates glucose tolerance. Diabetes 2005; 54:1009-1022.

18 Yechoor V, Liu V, Espiritu C, et al. Neurogenin3 is sufficient for transdetermination of hepatic progenitor cells into neo-islets in vivo but not transdifferentiation of hepatocytes. Dev Cell 2009; 16:358-373. 